\title{
The Role of Extracorporeal Shock Wave Therapy and Manual Lymphatic Drainage in Chronic Ulcers Treatment
}

\section{Raoul Saggini ${ }^{*}$, A Saggini ${ }^{2}$, Sm Carmignano ${ }^{3}$, T Palermo ${ }^{3}$, G Barassi $^{4}$, Mg Onesti $^{5}$, Rg Bellomo ${ }^{6}$ and N Scuderi $^{5}$}

${ }^{1}$ Full Professor, Physical and Rehabilitation Medicine, Department of Medical Oral and Biotechnological Sciences, Director of the School of Specialty in Physical and Rehabilitation Medicine, "Gabriele d'Annunzio" University, Chieti, Pescara, Italy National Coordinator of Schools of Specialty in Physical and Rehabilitation Medicine

${ }^{2}$ Anatomic Pathology, Department of Biomedicine and Prevention, University of Rome Tor Vergata, Rome, Italy

Dermatology, Department of Systems Medicine, University of Rome Tor Vergata, Rome, Italy

${ }^{3}$ School of Specialty in Physical and Rehabilitation Medicine, "Gabriele d'Annunzio" University, Chieti, Pescara, Italy

${ }^{4} \mathrm{PhD}$, LectureFaculty of Physiotherapy "G. d'Annunzio" University-Chieti, Italy

${ }^{5}$ Department of Plastic and Reconstructive Surgery, "Sapienza" University, Policlinico Umberto I, Rome, Italy

${ }^{6}$ Associated Professor, Physical and Rehabilitation Medicine, Department of Medicine and Sciences of Aging, "Gabriele d'Annunzio" University, Chieti, Pescara, Italy

\begin{abstract}
Introduction: Chronic wounds are a major, functionally-limiting medical problem impairing quality of life for millions of people each year. Extracorporeal shock wave therapy (ESWT) may accelerate and improve wound repair. Some authors investigated the optimal session number of ESWT in rat models. Aim: Purpose of this study was to verify the effectiveness of two temporal modalities of ESWT administration in chronic ulcers.

Materials and methods: 84 subjects with chronic ulcers were enrolled. They were divided into 2 groups according to the absence or presence of associated lymphedema. Each group was divided with a stratified randomization into two subgroups receiving the same total energy of ESWT ( $2640 \mathrm{Mj}$ ) but with different temporal modality during the week.

Results: Our results show, at the end of the 5 weeks treatment, a statistically significant reductionof wounds area and pain in the groups which received the ESWT fractioned in 2 weekly steps $(2 \times 1320 \mathrm{Mj})$ and reduction of limb circumference in subjects with lymphedema.

Discussion: By fractioning energy in 2 weekly steps probably there is greater regenerative activation. This type of application cannot be generalized, it could be a best choice in diabetic ulcers, even in the presence of lymphedema. The kwnoledge relative to the efficacy of ESWT in treatment of chronic wounds has had over the years a consolidation process, instead the modulation of time treatment can be considered as a new borders for clinical studies.
\end{abstract}

\section{Introduction}

A chronic wound can be defined as one that has failed to proceed through an orderly and timely reparative process to produce anatomic and functional integrity within a period of 3 months or that has proceeded through the repair process without establishing a sustained, anatomic and functional result [1]. They represent a complex and heterogeneous group of disorders whose incidence and prevalence varies per type of injury, care setting, age group and quality of care, but they are a frequent complication of diabetic disease [2].

Wound healing is a dynamic process influenced by homeostatic balance, inflammatory and matrix-synthesis process, and by an appropriate process of tissue remodelling. On a histological point of view, the healing process is divided into three main stages: inflammatory stage, proliferative stage, and maturation or re-modelling stage. The alteration of one of these physiological steps leads to the chronic wound. A chronic wound is histologically determined by lack of organization in endothelial proliferation, presence of parakeratotic keratinocytes, connective tissue disorganization, increasing in keratinocytes and granulocytes, disorders of proteins, electrolytes and cytokines [3,4]. The main causes affecting the physiological healing process are: pressure, slipping and shearing forces, reduced mobility, sensory-motor function impairment, poor nutrition, advanced age, changes in hematopoiesis and external factors such as psychosocial problems, prolonged immobilization and infections. About $70 \%$ of skin wounds are pressure ulcers, diabetic or vascular; other cases may

\section{Publication History:}

Received: October 05, 2016

Accepted: December 19, 2016

Published: December 21, 2016

\section{Keywords:}

Chronic ulcers, Lymphedema, Microcirculation, ESWT, Manual Lymphatic drainage, Repair be associated with inflammatory diseases, malignancy, burns or radiation damage [5]. The process of healing is influenced by the skin cells ability to respond to mechanical forces and their specific reaction is crucial to the way wounds behave in physical environment. It becomes more evident that mechanotransduction if appropriate can be a stimulus that improve tissue repair. Many studies suggest the importance of mechanical force transduction in wound healing process.It has been widely described in literature that shock waves are effective in stimulating several endogenous growth factors such as EGF, IGF1, VEGF and nitric oxide production, inducing angiogenesis and promoting the healing of fractures, ulcers and complex lesions. Furthermore, it was shown that the EWST regulates the activation of the pro and antifibrotic proteins (TGF- $\beta 1$ and matrix metalloproteinase 2 , respectively) that are involved in fibrosis: multiple sessions can reduce tissue fibrosis and promote the of reabsorption and / or remodeling processes [6-8].

"Corresponding Author: Prof. Raoul Saggini, Director of the School of Specialty in Physical and Rehabilitation Medicine, “Gabriele d'Annunzio" University, Chieti, Pescara, Italy; E-mail: saggini@unich.it

Citation: Saggini R, Saggini A, Carmignano SM, Palermo T, Barassi G, et al. (2016) The Role of Extracorporeal Shock Wave Therapy and Manual Lymphatic Drainage in Chronic Ulcers Treatment. Int J Clin Med Microbiol 3: 119. doi: https:// doi.org/10.15344/2456-4028/2016/119

Copyright: (c) 2016 Saggini et al. This is an open-access article distributed under the terms of the Creative Commons Attribution License, which permits unrestricted use, distribution, and reproduction in any medium, provided the original author and source are credited. 
Citation: Saggini R, Saggini A, Carmignano SM, Palermo T, Barassi G, et al. (2016) The Role of Extracorporeal Shock Wave Therapy and Manual Lymphatic Drainage in Chronic Ulcers Treatment. Int J Clin Med Microbiol 3: 119. doi: https://doi.org/10.15344/2456-4028/2016/119

Page 2 of 6

Few studies have investigated the timing of application of ESWT. Wang in 20099, investigated the optimal session number of ESWT in rat models. Stieger in 2013 10showed how the application of ESWT can improve the effect of lymphatic drainage in a non-healing chronic leg ulcer.

The purpose of this study was to verify the effectiveness of two temporal modalities of administration of ESWT and compare them to each other and to the combined therapy with manual lymphatic drainage in presence of lymphedema.

\section{Materials And Methods}

The study was performed by the Department of Physical Medicine and Rehabilitation of "G. D'Annunzio" University in Chieti, and the Department of Plastic and Reconstructive Surgery of "La Sapienza" University in Rome; it was approved by the local ethics committee, and performed in accordance with the 1964 Declaration of Helsinki.

103 subjects had come to our attention.Inclusion criteria were: age between 18 and 70 years old, diagnosis of diabetic foot syndrome, wounds not healing within 3 months in spite of correct management, Wagner's classification grade 3 or less 11 ;exclusion criteria: patients with neoplasticor neurodegenerative diseases, lymphedema grade 4 or above12, Mini-Mental State Examination, $($ MMSE) $<26$.

15 subjects do not respect the inclusion criteria and 4 patients were excluded for absence of written consent. Therefore 84 patients (47

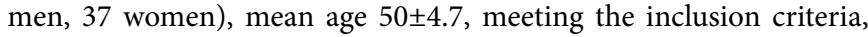
were evaluated and treated after acceptance of informed consent (figure 1).
All patients were suffering from diabetes and they went to our attention with the diagnosis of diabetic foot syndrome. They were divided into 2 groups (A and $\mathrm{B}$ ) of 42 subjects according to the absence or presence of associated lymphedema, diagnosed by clinical examination and duplex sonography. Each group was then further divided into two subgroups through a stratified randomizationwith regard to 3 prognostic factors, smoking status, degree of ulceration, degree of lymphedema in order to have homogeneous groups. Each one made of 21 subjectswho made ESWT at different weekly frequency.All subjects were treated with Dermagoldelectroidraulic device equipped with an unfocused probe (MTS Europe GmbH, Constance, Germany).

Patients in group A-Ireceived 1 session per week of ESWT for 5 weeks ( 1000 pulses were administered for each session), yet thegroup A-II received 2 session per week (every 84 hours) of ESWT for 5 weeks (500 pulses for each session).

Subjects in groups B, affected by lymphedema, received: 1 session per week of ESWT for 5 week (1000 pulses for each session) for group B-I and 2 sessions per week (every 84 hours) of ESWT for 5 weeks (500 pulses for each session) for group B-II; manual lymphatic drainage was also performed in bothsubgroupsB according to their underlaying disease.

The mean energy density applied for each pulse was $0.13 \mathrm{~mJ} / \mathrm{mm} 2$ in all groups.

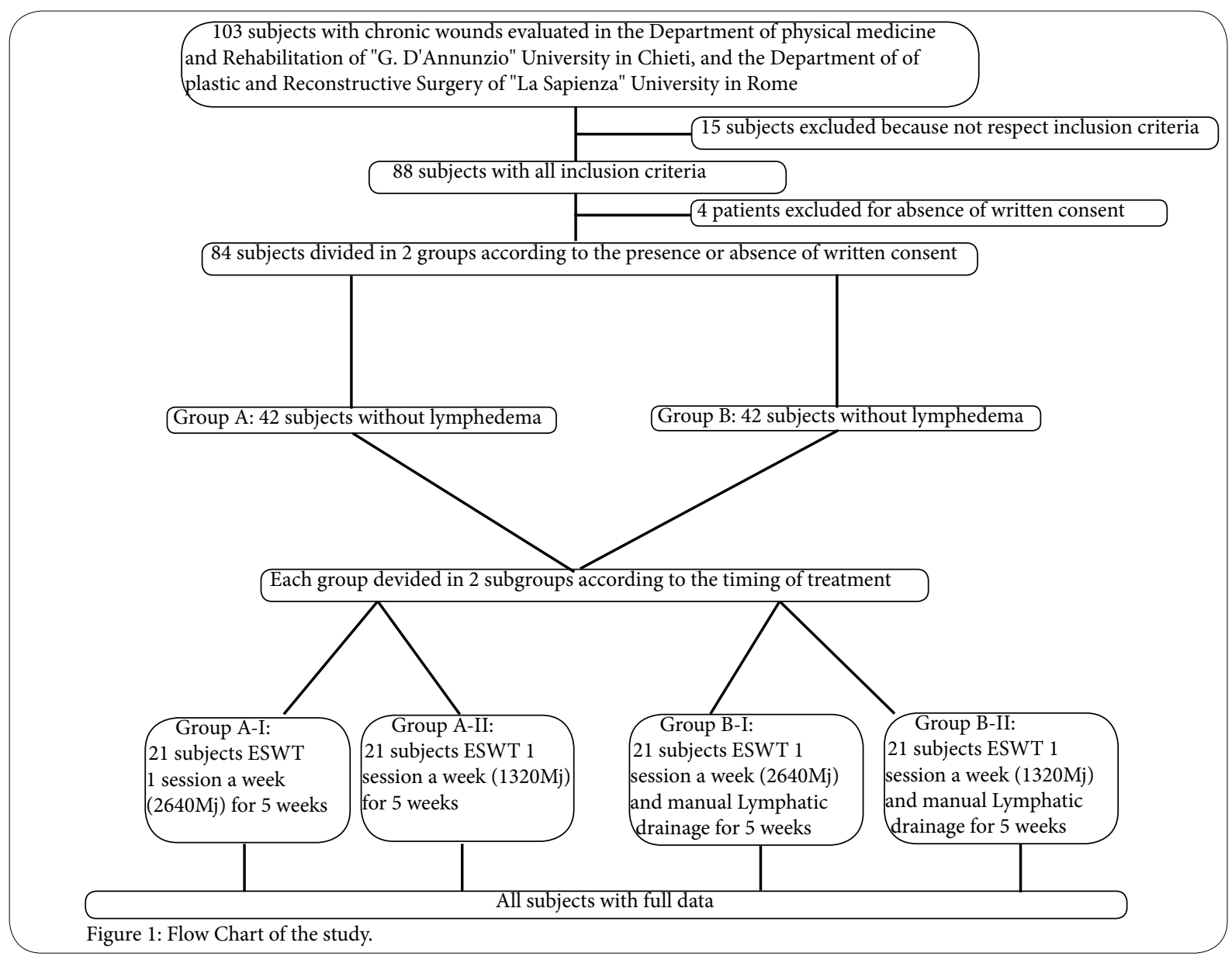


Citation: Saggini R, Saggini A, Carmignano SM, Palermo T, Barassi G, et al. (2016) The Role of Extracorporeal Shock Wave Therapy and Manual Lymphatic Drainage in Chronic Ulcers Treatment. Int J Clin Med Microbiol 3: 119. doi: https://doi.org/10.15344/2456-4028/2016/119

Before and after treatment period ulcers were classified according to: localization, width (in $\mathrm{cm}$ ), length (in $\mathrm{cm}$ ), percentage of granulation tissue, necrotic tissue, fibrous tissue, presence of bacterial exudate (classified as absent, minimal, moderate, high) and pain, which have been assessed by VAS (Visual Analogic Scale). Lymphedema was evaluated by ankle circumferenceat $\mathrm{T} 0$ and $\mathrm{T} 1$.

The morphological evolution was monitored taking photos before each session using digital cameras with resolution higher than 5 megapixels and macros function; all pictures were taken at the shortest distance necessary to frame the lesion and the standardized squared. After each session a medication with povidone-iodine, Amukine MED $0.05 \%$ and application of semipermeable occlusive dressing was performed; this protocol was identically in all groups and in every session.

No compressive bandage was applied to the patientsduring the treatment period and was also monitored the possible occurrence of side effects such as pain, petechiae and cutaneous adverse reactions related to ESWT therapy. Lymphedema in groups B-I and B-II was evaluatedat $\mathrm{T} 0$ and $\mathrm{T} 1$ by measuring the ankle circumference with a standard tape measure (MediGmbh, Bayreuth,Germany) [12].

All data are given as means \pm SDs. Differences between mean values before and after the rehabilitation period were tested for significance using Student's t-test for paired observations. The minimum level of statistical significance was set at $\mathrm{p}<0.05$.

\section{Results}

Before treatment (T0) in group A-I the mean wound dimension was $1.87 \pm 0.53 \mathrm{~cm}^{2}$ and the mean VAS score for subjective pain was $5.7 \pm 1.47$; in group A-II the mean wound dimension at the first evaluation (T0) was $1.69 \pm 0.52 \mathrm{~cm}^{2}$ and the mean VAS score was $5.1 \pm 1.13$; in group B-I the mean wound area at T0 was $2.83 \pm 0.97 \mathrm{~cm}^{2}$ and the mean perceived subjective pain was $5.5 \pm 1.54$; in group B-II before treatement (T0) the mean wound size was $2.71 \pm 0.68 \mathrm{~cm}^{2}$ and the mean VAS score for subjective pain was $5.3 \pm 1.40$.

At the end of the 5 weeks treatment (T1) we found:

In group A-I a reduction of wound area from $1.87 \pm 0.53 \mathrm{~cm}^{2}(\mathrm{~T} 0)$ to $0.77 \pm 0.34 \mathrm{~cm}^{2}(\mathrm{p}<0.01)$ and a reduction of the perceived subjective pain from $5,7 \pm 1.47$ (T0) to $1.8 \pm 0.72$ ( $\mathrm{p}<0.01$ ); In group A-II a reduction of wound size from $1.69 \pm 0.52 \mathrm{~cm}^{2}$ (T0) to $0.45 \pm 0.13 \mathrm{~cm}^{2}(\mathrm{p}<0.001)$; a reduction of VAS score from $5,1 \pm 1.13$ (T0) to $0.5 \pm 0.11(\mathrm{p}<0.001)$

In group B-I a reduction of wound area from $2.83 \pm 0.97 \mathrm{~cm} 2$ (T0) to $1.88 \pm 0.59 \mathrm{~cm}^{2}(\mathrm{p}<0.05)$; a reduction of the perceived pain from $5,5 \pm 1.54(\mathrm{~T} 0)$ to $2.9 \pm 1.31(\mathrm{p}<0.05)$; In group B-II a reduction of wound dimensions from $2.71 \pm 0.77 \mathrm{~cm}^{2}$ (T0) to $1.54 \pm 0.23 \mathrm{~cm}^{2}(\mathrm{p}<0.01)$; a reduction of the perceived subjective pain from $5,3 \pm 1.40$ (T0) to $2.3 \pm 0.47(\mathrm{p}<0.001)$

We found a significant reduction of wound area and VAS score in all groups (figures 2 and 3 ).

Although there were significant final data in all groups, the best results, in terms of absolute value, were found in groupA-II and B-II, which performed ESWTtwice a week with 500 pulses per session. No side effects were found during treatment.

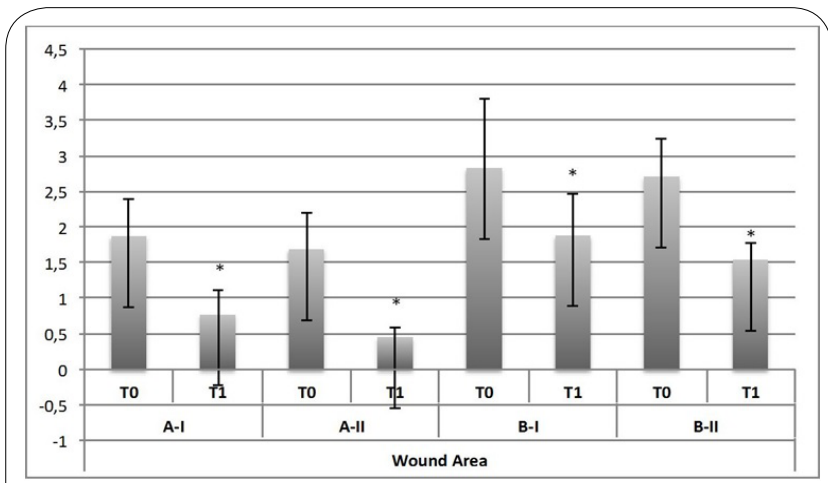

Figure 2: shows the reduction in wound area after the treatment period in the 4 groups; ${ }^{*}=\mathrm{p}$ value $<0.05$.

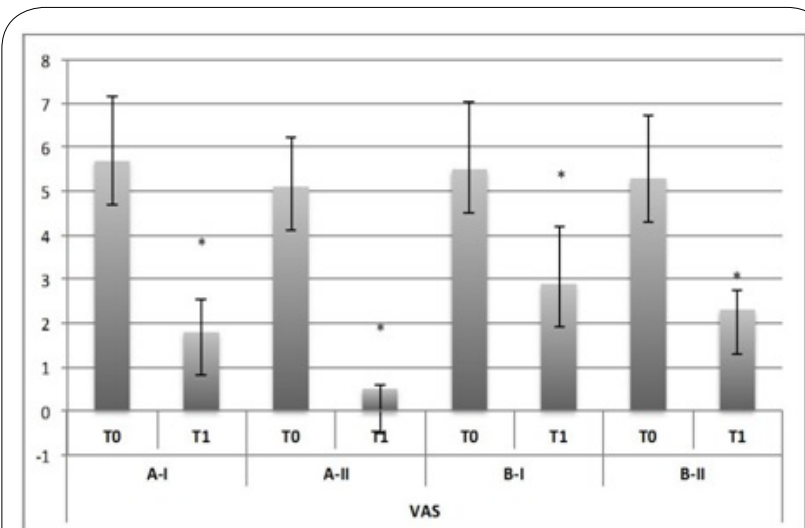

Figure 3: shows the trend of pain before and after the treatment period; ${ }^{*}=\mathrm{p}$ value $<0.05$.

In groups B-I and B-II,whose subjects were affected by lymphedema, we found a reduction of limb circumference. The initial mean circumference of lower limb assessed on the ankle was $30.7 \pm 3.1$ cmin group B-I and $31.1 \pm 2.9 \mathrm{~cm}$ in group B-II. Thefinal result was significant only in group B-II which performed the treatment 2 times a week: $3.4 \%$ (final circumference $29.7 \mathrm{~cm}$ ) in group B-Iand 5.8 $\%$ (final circumference $29.3 \mathrm{~cm}$ ) in group B-II $(\mathrm{p}<0.05)$ (figure 4 ).

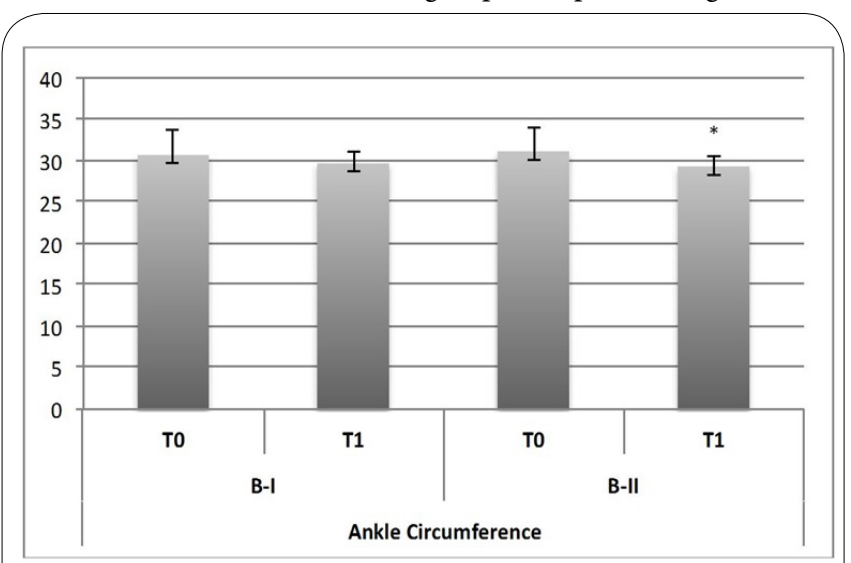

Figure 4: shows the measurement of ankle circumference in the two groups with lymphedema, ${ }^{*}=$ p value $<0.05$.

\section{Discussion}

Wound duration is a quantifiable surrogate for one or more undefined variables that can have a profound negative effect on ulcer healing [13]. Has been widely described how the venous system damage, 
Citation: Saggini R, Saggini A, Carmignano SM, Palermo T, Barassi G, et al. (2016) The Role of Extracorporeal Shock Wave Therapy and Manual Lymphatic Drainage in Chronic Ulcers Treatment. Int J Clin Med Microbiol 3: 119. doi: https://doi.org/10.15344/2456-4028/2016/119

Page 4 of 6

destruction of microlymphatics and local nerve fibers lead to impaired fluid drainage and altered regulatory mechanisms, resulting in impairment of wound healing. Comorbid conditions of diabetes such as obesity, venous insufficiency and rheumatoid arthritis can also interfere with a proper wound-healing response [14].

Several studies showed how ESWT can be able to accelerate wound healing reducing time for cicatrization, promoting the rearrangement of endothelial cells and the basal lamina with a significant increase in the release of growth factors, nitric oxide, TGF- $\beta 1$ and nuclear proliferation antigen 15,16 ,by inducing mechanostransduction; they can also reduce the incidence of infections thanks to the bactericidal effect and reduce pain by inducing degeneration of the nerve fibers originating from small neurons ATF3-immunoreactive, which seems to achieve a relief of pain and influence the increase in Substance P $[17,18]$.

According to Stieger [10], which showed an effect of ESWT on limphatic drainage in non-healing chronic leg ulcers, we applied the unfocused Shock Wave treatment on chronic leg ulcers even in case of lymphedema due to the specific wound. Stieger results could be linked with the cavitation effect of ESWT on tissues since the reflection wave induced by the device triggers a jet stream microcavitational resulting in increased tissue perfusion (as shown by Wang et al. [9] in a rat model), reduction of the local inflammatory reaction with upregulation of cell proliferation, particularly of fibroblasts and keratinocytes, and angiogenesis, through an increased expression of VEGF ("Vascular Endothelial Growth Factor")and eNOS (endothelial Nitircoxidsintethase)

Recently, regeneration of lymphatic vessels has been demonstrated by using physical treatment methods such as extracorporeal shockwave therapy (ESWT) [19], EWST leads to increased cell permeability and expression of growth factors, such as VEGF-C, which is associated with promoting lymphangiogenesis and accelerating capillary morphogenesis [20,21]; Kim et al. used mice suffering from lymphedema at the mid-thigh to investigate the effect of gelatine hydrogels containing VEGF-C and/or ESWT. The combination of treatment methods (VEGF-C hydrogels and ESWT) showed the best results in terms of lymphatic vessel formation, improvement of lymphedema, and enhanced expression of VEGF-C and VEGFR-3 [22].

ESWT increases the platelet-cell adhesion molecule-1 (PECAM-1) production on leukocytes and on endothelial cells which mediate a physical link between the cell surface and the nuclear envelope. It is critically involved in the trans-endothelial migration processes at inflammatory sites, endothelial cell migration, and formation of new blood vessels. This may transmit mechanical orbiochemicalsignalsthat may modulate the genic expression of cells $[23,24]$.

Schaden et al. [25] and Mariotto et al. [26] demonstrated that ESWT can promote angiogenesis, decrease neutrophils and inflammation, and decrease the number of adipocytes.

Wang at al, 2014 [27] evaluated the long-term effects of ESWT in chronic foot in a long follow up during 5 years. The evaluations included clinical assessment of the ulcer status, including the size, shape, and depth with photo documentation, local blood flow perfusion scan, and the mortality and morbidity (including amputation) in 1 and $5 \mathrm{y}$ after ESWT. Tissue viability was evaluated by local blood flow perfusion scan preoperatively and at $6 \mathrm{wk}, 1 \mathrm{y}$, and $5 \mathrm{y}$ postoperatively.
After ESWT, the blood perfusion rates is greatly improved in both DM $(\mathrm{p}=0.011)$ and non-DM $(\mathrm{p}=0.033)$ groups. The flow perfusion rate was better after 6 weeks, and the improvement was maintained for one year. In both groups, the percentage of blood perfusion decreased in the follow up to 5 years. Non-DM group showed a better blood flow perfusion than that of the DM groupat 1 year to 5 years follow up.

The clinical outcomes, mortality, and morbidity were compared with a database control group of 149 patients with diabetic foot ulcers previously treated by the author. The experimental group showed better results than the data with no significant differences respect the control database. Complications such as amputations were higher in the control group at both one year than five years. The mortality rate was $9.4 \%$ in historical controls and $0 \%$ to 1 year and $24 \%$ to 5 years in the experimental ESWT patients. At the conclusion of this study Wang et al concluded that ESWT appears effective in the treatment of chronic diabetic and non diabetic foot ulcers. However, the effects of ESWT significantly decreased from 1 to $5 \mathrm{y}$ after treatment.

The study included 38 patients with 40 ulcers in the diabetes mellitus (DM) group and 29 patients with 32 ulcers in the non-diabetes mellitus (non DM) group. All patients received unfocused ESWT Few studies have investigated the timing of application of ESWT.

Wang in 2009 [9] investigated the ideal frequency of administration and the bio-mechanisms operating during ESWT of wounds in diabetic wounds treatment in rats models. Fifty male Wistar rats were divided into five groups. GroupI consisted of non-diabetic control; group II included diabetic control receiving no ESWT; group III included rats that underwent one session of ESWT (ESW-1) on day 3 (800 impulses at $0.09 \mathrm{~mJ} / \mathrm{mm}$ (2) post-wounding; group IV included rats that underwent two sessions of ESWT (ESW-2) on days 3 and 7; and group $\mathrm{V}$ included rats that underwent three sessions of ESWT (ESW-3) on days 3, 7, and 10. The number of pulses (800) was the same for each session. The wound healing was assessed clinically. Blood perfusion scan was performed with laser Doppler. The VEGF, eNOS, and PCNA were analyzed with immunohistochemical stain. The results revealed that the wound size was significantly reduced in the ESWT-treated rats, especially in the ESW-2 and ESW-3 groups, which performed the treatment with a higher frequency during the week, as compared to the control. Blood perfusion was significantly increased after ESWT compared to the controls. Histological findings revealed a significant reduction in the topical pro-inflammatory reaction in the ESWT group as compared to the control. In immunohistochemical stain, significant increases in VEGF, eNOS, and PCNA expressions were observed in the ESWT group, especially in the ESW-2 and ESW-3 groups, as compared to the control. So he concluded that the treatment with an optimal session of ESWT performed twice or three times a week could significantly enhance diabetic wound healing; this effect was strictly associated with the increase in neo-angiogenesis and tissue regeneration, and topical anti-inflammatory response.

Furthermore Bae in 2013 [21] showed how ESWT is an effective modality in the treatment of stage 3 lymphedema after breast cancer treatment, leading to a reduction of the circumference and thickness of arms with lymphedema providing clinically favorable outcome to patients. In his studyESWT was carried out twice a week for two weeks using electromagnetic type and the stimulus was given on the treatment site according to the patient's tolerance, 2,000 times in one session with an energy of $0.056-0.068 \mathrm{~mJ} / \mathrm{mm}^{2}$. Stimulus was applied 1,000 times to the most fibrotic lesion felt by the examiner's palpation, and the other 1,000 times was applied to other less fibrotic lesion. CDPT (Complex Decongestive Physical Therapy) or pneumatic 
Citation: Saggini R, Saggini A, Carmignano SM, Palermo T, Barassi G, et al. (2016) The Role of Extracorporeal Shock Wave Therapy and Manual Lymphatic Drainage in Chronic Ulcers Treatment. Int J Clin Med Microbiol 3: 119. doi: https://doi.org/10.15344/2456-4028/2016/119

Page 5 of 6

compression was not successful on these subjects in the past: so he divided subjects in two groups by their opinion. 4 patients were treated by manual lymphatic massage and pneumatic compression with ESWT; other 3 patients were treated with ESWT only. He found a significant reduction in lymphedema volume, limb circumference, and skin fold thickness in all subjects who were treated with four sessions of ESWT, concluding that ESWT can be considered an effective modality in the treatment of stage 3 lymphedema after breast cancer operation, with or without other traditional treatment modalities.

Christ et al. [28] confirmed the improvement of skin elasticity in the treatment of cellulitis and connective tissue weakness by means of ESWT. Patients in their study received treatment for 6 therapy sessions and were followed up for 3 months. It was observed that the network of collagen/elastic fibers in the dermis and subcutaneous became denser and measurably firmer. In the parallel biomechanical examinations, reduced oxidative stress by ESWT was shown by this study by means of increased lipolysis and by the release of toxic aldehydic products of lipid; furthermore they showed the improvement in collagen synthesis and in skin condition. This study also confirmed the decrease of orange pill sign, which was shown in stage 3 lymphedema, and other measurements of skin conditions including skin fold thickness and skin hardness. This may indicate improvement of skin elasticity and connective tissue strength.

Tinazzi et al. [29] applied ESWT to patients affected by systemic sclerosis showing an improvement of VAS and Rodnan skin score for skin wellness and in increased endothelial progenitor cells and circulatory endothelial cells.

According to Wang [9] we administered the same therapy and the same number of pulses in all groups of study, but with different temporal modalities during the week, by splitting in two times (500 every 84 hours instead of 1000 in a single session) the amount of pulses administered in groups A-II and B-II. So 1000 pulses one time a week were administered in groups A-I (chronic wound) and B-I (chronic wound and lymphedema) and 500 pulses two times a week were administered in goups A-II (chronic wound) and B-II (chronic wound and lymphedema). Results showed how ESWT have been significantly able to improve healing in all groups of treatment, but with a greater result as for absolute values in those groups (AII and B-II) which performed the treatment twice a week. In group B-II, which had concomitant lymphedema and performed the treatment twice a week, we also found a significant reduction of ankle circumference.

\section{Conclusion}

Considering all this evidences, we can state that the ability of ESWT to act on the repair of microvascular damage in chronic wounds induces, on the one hand the activation of the skin repair process and on the other the recovery of adequate venous and lymphatic microcirculation. In addition, ESWT induces an improvement in blood flow and therefore reduces edema associated with chronic wound, even in the absence of compression bandage.

Particularly, in the skin healing mode processes by which cells react to mechanical forces it is crucial in the switch between healing, failure to re-epithelialization and hypertrophic scar. In vitro studies show that the mechanical stimulus induced by ESWT in metabolic processesthat involve the production of fibroblasts and keratinocytes is able to lead the process towards the physiological repair of the wound.
Mechanical signals received by fibroblasts guide their transformation from a soft substrate with a little adhesion and poorly developed stress fibers into an adherent strongly geometry, that improves keratinocyte differentiation [7].

The intracellular mechanical tension and the conversion of mechanical signals in biomolecular events, related to the expression of specific genes, are responsible for the contractile force and the creation of a good environment for reparative and regenerative processes, especially when it is associated with the presence of lymphedema.

The progressive pathologic evolution, in fact, related to interstitial lymphatic hypertension and the consequent tissue acidosis, hypoxia, accumulation of lymphocytes, of free radicals and other toxic substances, slowing the healing process. Furthermore, low-energy ESWT have been proven to induce therapeutic lymphangiogenesis by up-regulating vascular endothelial growth factor $\mathrm{C}$ and basis fibroblast growth factor, and by improving lymphedema. Therefore, the improvement of lymphedema in our study could be the result of lymphangiogenesis. The change in lower limb circumference in this study could be the result of both manual lymphatic drainage and ESWT promoting reduction of inflammatory cells, reconditioning of skin tissue and improvement of lymphatic drainage.

Based on our clinical experiences and researches related to the effect of ESWT on the healing of chronic wounds 6,30 and the effect of the manual drainage in aiding the healing processes [31,32]we believe that the most appropriate treatment time is 2 sessions weekly with total energy of $2640 \mathrm{Mj}$ (which it is the average energy applied in our previous studies and experiences of six years of work on chroniculcers) divided in two weekly applications spaced from each other by 84 hours $(2 \times 1320 \mathrm{Mj})$ rather than in a single administration $(1 \times 2640 \mathrm{Mj})$ in order to keep the healing promoting stimulus without a stimulus deflection phase during the week.

This type of application can not be generalized, it could be probably a best choice for chroniculcers in presence of lymphedema.

The results obtained in subjects with lymphedema, in which the presence of edema represents a delay element for healing, can encourage us in confirming that the healing process is based on angiogenesis, that is very important to promote drainage, but above all to confirm that shock waves are crucial in reactivating and accelerating the healing process of chronic wounds.

Limitations of the study is the absence of a comparative group (with the same standard treatment but without ESWT); further investigation with a larger sample will be necessary to consolidate our results.

\section{Competing Interests}

The authors declare that they have no conflict of interest in this work.

\section{References}

1. Järbrink K, Ni G, Sönnergren H, Schmidtchen A, Pang C, et al. (2016) Prevalence and incidence of chronic wounds and related complications: a protocol for a systematic review. Syst Rev 5: 152.

2. Hingorani $A$, La Muraglia GM, Henke $P$, Meissner $M H$, Loretz $L$ et al. (2016) The management of diabetic foot: a clinical practice guideline by the Society for Vascular Surgery in collaboration with the American Podiatric Medical Association and the Society for Vascular Medicine. J Vasc Surg 63: 3S-21S. 
Citation: Saggini R, Saggini A, Carmignano SM, Palermo T, Barassi G, et al. (2016) The Role of Extracorporeal Shock Wave Therapy and Manual Lymphatic Drainage in Chronic Ulcers Treatment. Int J Clin Med Microbiol 3: 119. doi: https://doi.org/10.15344/2456-4028/2016/119

3. Kasuya A, Tokura Y (2014) Attempts to accelerate wound healing. J Dermatol Sci 76: 169-172.

4. Turi GK, Donovan V, DiGregorio J, Criscitelli TM, Kashan B, et al. (2016) Major Histopathologic Diagnoses of Chronic Wounds. Adv Skin Wound Care 29: 376-382.

5. Kuo YR, Wang CT, Wang FS, Chiang YC, Wang CJ (2009) Extracorporea shock-wave therapy enhanced wound healing via increasing topical blood perfusion and tissue regeneration in a rat model of STZ-induced diabetes. Wound Repair Regen 17: 522-530.

6. Saggini R, Figus A, Troccola A, Cocco V, Saggini A, et al. (2008) Extracorporeal shock wave therapy for management of chronic ulcers in the lower extremities. Ultrasound Med Biol 34: 1261-1271.

7. Rosi A, zuk J, Taradaj J, Dymarek R, Sopel M (2016) Mechanoregulation of Wound Healing and Skin Homeostasis. Biomed Res Int 2016: 3943481.

8. D'Agostino MC, Craig K, Tibalt E, Respizzi (2015) Shock wave as biological therapeutic tool: From mechanical stimulation to recovery and healing, through mechanotransduction. Int J Surg 24: 147-153.

9. Moayednia A, Haghdani S, Khosrawi S, Yousefi E, Vahdatpour B (2014) Long-term effect of extracorporeal shock wave therapy on the treatment of chronic pelvic pain syndrome due to non bacterial prostatitis. J Res Med Sci 19: 293-296.

10. Stieger M, Schmid JP, Bajrami S, Hunziker T (2013) [Extracorporeal shock wave therapy as a treatment of a non-healing chronic leg ulcer]. Hautarzt 64: $443-446$

11. Wagner FW Jr (1986) The diabetic foot and amputation of the foot, In Surgery of the Foot, (Mann, ed.) Mosby, St Louis, pp. 421- 455.

12. Arin KG, Slavin SA,Håkan B (2015) Lymphedema Presentation, Diagnosis, and Treatment. Springer International Publishing Switzerland

13. Frykberg RG, Gibbons GW, Walters JL, Wukich DK, Milstein FC (2016) A prospective, multicentre, open-label, single-arm clinical trial for treatment of chronic complex diabetic foot wounds with exposed tendon and/or bone: positive clinical outcomes of viable cryopreserved human placental membrane. Int Wound $J$.

14. Lantis JC, Marston WA, Farber A, Kirsner RS, Zhang Y, et al (2013) The influence of patient and wound variables on healing of venous leg ulcers in a randomized controlled trial of growth-arrested allogeneic keratinocytes and fibroblasts. J Vasc Surg 58: 433-439.

15. Finlayson K, Edwards H, Courtney M (2009) Factors associated with recurrence of venous leg ulcers: a survey and retrospective chart review. Int J Nurs Stud 46: 1071-1078.

16. Dymarek R, Halski T, Ptaszkowski K, Slupska L, Rosinczuk J, et al. (2014) Extracorporeal shock wave therapy as an adjunct wound treatment: systematic review of the literature. Ostomy Wound Manage 60: 26-39.

17. Huemer GM, Meirer R, Gurunluoglu R, Kamelger FS, Dunst KM, et al. (2005) Comparison of the effectiveness of gene therapy with transforming growth factor-beta or extracorporal shock wave therapy to reduce ischemic necrosis in an epigastric skin flap model in rats. Wound Repair Regen 13: 262-268.

18. Holfeld J, Tepeköylü C, Kozaryn R, Urbschat A, Zacharowski K, et al (2014) Shockwave Therapy Differentially Stimulates Endothelial Cells: Implications on the Control of Inflammation via Toll-Like Receptor 3. Inflammation 37: S65-S70.

19. Schaupper M, Jeltsch M, Rohringer S, Redl H, et al. (2016) Lymphatic Vessels in Regenerative Medicine and Tissue Engineering. Tissue Eng Part B Rev 22: 395-407.

20. Kubo M, Li TS, Kamota T, Ohshima M, Shirasawa B, et al. (2010) Extracorporeal shock wave therapy ameliorates secondary lymphedema by promoting lymphangiogenesis. J Vasc Surg 52: 429-434.

21. Bae H, Kim HJ (2013) Clinical outcomes of extracorporeal shock wave therapy in patients with secondary lymphedema: a pilot study. Ann Rehabi Med 37: 229-234.

22. Kim IG, Lee JY, Lee DS, Kwon JY, Hwang JH (2013) Extracorporeal shock wave therapy combined with vascular endothelial growth factor-C hydroge for lymphangiogenesis. J Vasc Res 50: 124-133.

23. Uriu K, Morelli LG, Oates AC (2014) Interplay between intercellular signaling and cell movement in development. Semin Cell Dev Biol 35: 66-72.
24. Dupont S (2016) Role of YAP/TAZ in cell-matrix adhesion-mediated signalling and mechanotransduction. Exp Cell Res 343: 42-53.

25. Schaden W, Thiele R, Kölpl C, Pusch M, Nissan A, et al. (2007) Shock wave therapy for acute and chronic soft tissue wounds: a feasibility study. J Surg Res 143: 1-12.

26. Mariotto S, Cavalieri E, Amelio E, Ciampa AR, de Prati AC, et al. (2005) Extracorporeal shock waves: from lithotripsy to anti-inflammatory action by NO production. Nitric Oxide 12: 89-96.

27. Wang CJ, Wu CT, Yang YJ, Liu RT, Kuo YR (2014) Long-term outcomes of extracorporeal shockwave therapy for chronic foot ulcers. J Surg Res 189: 366-372.

28. Christ C, Brenke R, Sattler G, Siems W, Novak P, et al. (2008) Improvement in skin elasticity in the treatment of cellulite and connective tissue weakness by means of extracorporeal pulse activation therapy. Aesthet Surg J 28: 538-544.

29. Tinazzi E, Amelio E, Marangoni E, Guerra C, Puccetti A, et al. (2011) Effects of shock wave therapy in the skin of patients with progressive systemic sclerosis: a pilot study. Rheumatol Int 31: 651-656.

30. Saggini R, Fioramonti P, Bellomo RG, Di Stefano A, Scarcello L, et al. (2013) Chronic ulcers: treatment with unfocused extracorporeal shock waves. European Journal of Inflammation11: 99-509.

31. Bellomo RG, Barassi G, Lococo A, Carmignano SM, Di lulio A, et al. (2015) Rehabilitation of cancer pain in lung cancer: role of manual therapy. Arch Physiother Glob Res 19: 27-38.

32. Saggini R, Barassi G, Di Felice PA, Frasci R, Dodaj I, et al. (2014) Integrated treatment with endodermal massage, proprioceptive rehabilitation, viscoelastic, plantar orthosis and myofascial manual therapy on skin graft: case report. Arch Physiother Glob Res 18: 23-34 